\title{
Analytical Modeling and Experimental Assessment of Five-Dimensional Capacitive Displacement Sensor
}

\author{
Jian-Ping Yu, ${ }^{*}$ Wen Wang, ${ }^{1}$ Xin Li, and Zhao-Zhong Zhou \\ College of Mechanical Engineering, Quzhou University, Quzhou, Zhejiang Province, China \\ ${ }^{1}$ School of Mechanical Engineering, Hangzhou Dianzi University, Hangzhou, Zhejiang Province, China
}

(Received April 19, 2016; accepted March 22, 2017)

Keywords: five-dimensional, capacitive sensor, position, rotational disturbances

This paper presents studies on a capacitive incremental displacement microsensor particularly for micro/nanopositioning applications. An incremental capacitive microsensor is capable of achieving large-scale, high-precision $X-Y$ linear positioning; however, some inevitable static errors and dynamic disturbances in reality affect the linearity of the $X-Y$ signal in the form of roll, yaw, and pitch movements. To realize high-precision $X-Y$ linear positioning, a symmetrical sensor modeling scheme and a novel signal processing scheme are developed to compensate for signal nonlinearity caused by rotational disturbances. At the same time, roll, yaw, and pitch signals are decoupled from $X-Y$ linear signals for possible feedback control purposes. A printed circuit board microsensor prototype for testing is constructed with a design featuring a $20 \mathrm{~mm}$ linear stroke, a $2 \mathrm{~mm}$ electrode pitch, and a $0.5 \mathrm{~mm}$ gap distance. The measured $X-Y$ signal nonlinearity is decreased to $0.5 \%$ with a $4 \mathrm{~mm}$ stroke, while signal errors of rotational disturbances are no larger than $0.01^{\circ}$. The feasibility of a five-dimensional displacement measurement, including a large stroke, the high-precision acquisition of $X-Y$ linear displacement, and roll, yaw, and pitch movements, is experimentally validated.

\section{Introduction}

During the past few decades, precise micro/nanopositioning has been required during the processes of micromachining and manipulation, such as in the fabrication of optical devices and semiconductors, cell manipulation, portable probe storage systems, and others. ${ }^{(1-4)}$ For better control of stability, a typical precise positioning test bench for these applications is usually a prototype with multidegrees of freedom. The conventional scheme of realizing measurements with multidegrees of freedom involves the stacking of multiple single-axis positioning systems, which results in a bulky and complicated structure involving unpredictable abbe errors and cumulative misalignment errors. To overcome these shortcomings, a planar positioning system as an alternative to achieve measurements with multi-degrees of freedom has been proposed.

Interferometers are usually used as measuring probes for a planar positioning system, because they offer the advantage of high resolution, but they suffer from bulkiness and sensitivity to measurement conditions such as air pressure, temperature, and humidity. ${ }^{(1,2)}$ Planar positioning systems can also be patterned in capacitor mechanisms. Huang et al. presented a microcapacitive 
position sensor for nanopositioning applications, which provided two linear position signals and one rotational position signal. ${ }^{(4)}$ The linear stroke was limited to $300 \mu \mathrm{m}$ peak-to-peak. ${ }^{(3)}$ Ahn and Jeon proposed a new smart sensor system that can measure five-dimensional motions. ${ }^{(1)}$ However, this sensor structure was mainly applied to a rotating machinery. ${ }^{(4)}$ Although capacitive sensing arrays may suffer from external noise, the capacitive approach still has some intrinsic advantages, such as fast response, high repeatability, and insensitivity to temperature variation.

The authors have developed a planar capacitive sensor capable of two-dimensional measurement. The interpretation of displacement depends on the results of measuring the periodic variation in capacitance caused by the change in the overlapping area of sensing electrodes. However, the main shortcomings of the proposed sensor are the impact from static errors (mainly as misalignment errors) and dynamic disturbances (kinematic and vibration errors) in the forms of sensitivity differences and phase-shift errors.

This paper presents an improved capacitive incremental displacement microsensor. To achieve high-precision $X-Y$ linear positioning, a symmetrical sensor modeling scheme and a phase-shift arctangent processing scheme were developed to compensate for signal deformations caused by rotational disturbances, while at the same time, three rotational signals were decoupled from $X-Y$ signals for latter feedback control purposes.

This paper is organized as follows. A complete description of the basic sensor structure is presented in Sect. 2. In Sect. 3, the sensor mode is analyzed with respect to harmonic errors. In Sect. 4, a novel scheme of five-dimensional displacement measurements is proposed for enhancing linearity and deriving three rotational signals. After a detailed description of the sensor system prototype in Sect. 5, extensive experimental results are presented.

\section{Basic Sensor Structure}

The basic sensor structure of a typical capacitive planar positioning system is illustrated in Fig. 1. The sensor has a periodic electrode pattern on both a moving plate (MP) and a fixed plate (FP) to maximize capacitance change. In the figure, $w$ is the common electrode width, $l$ is the sensing electrode length, $g$ is the gap distance, and $L$ is the vertical distance from the sensing electrodes to the assumed center.

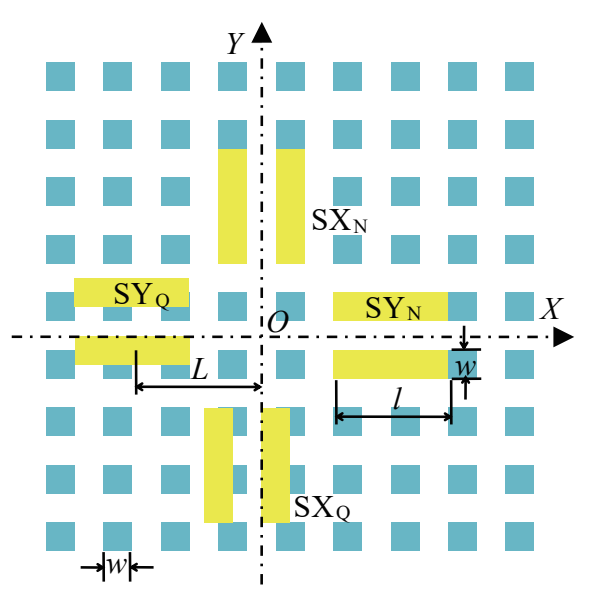

Fig. 1. (Color online) Basic sensor structure of a typical capacitive planar positioning system. 
Furthermore, there is a pair of normal and quadrature sensing electrodes on the FP for both $X$ and $Y$ displacement demodulations, namely, $\mathrm{SX}_{\mathrm{N}}, \mathrm{SX}_{\mathrm{Q}}, \mathrm{SY}_{\mathrm{N}}$, and $\mathrm{SY}_{\mathrm{Q}}$. Setting aside fringe effects, signals from sensing electrodes are all in perfect periodic triangular waveforms given that the common electrodes on the MP are parallel. However, in reality, fringe effects would introduce a severe uncertain distortion to signal waveforms. To clarify the fringe effects on signal nonlinearity, a full capacitance model was calculated firstly using Maxwell's equation: ${ }^{(5)}$

$$
C(x)=\frac{Q}{V_{\text {input }}}=-\varepsilon_{0} \varepsilon_{r} w\left\{\frac{w}{2 g}-\sum_{n=\text { odd }} \frac{4}{k_{n} w \sinh \left(k_{n} g\right)} \cos \left(k_{n} x\right)\right\},
$$

where $k_{n}=\frac{n \pi}{w}$.

As in Eq. (1), electrode width is implied to be a key factor that determines the ratio of sensor waveform nonlinearity to gap distance $(w / g)$. Figure 2 illustrates a comparison of the standard deviations of the waveform deformation errors of the proposed sensor signals to ideal triangular and sinusoidal waveforms for different $w / g$ ratios. The comparison indicates that the actual waveform is more closely dependent on sinusoidal waveforms. Thus, in the actual sensor model, signals are considered sinusoidal waveforms. Nevertheless, an improvement in modeling can be attempted using a numerical approach and a more elaborate curve fitting. Based on these conclusions, both $X$ and $Y$ positions are retrieved by a standard arctangent operation.

$$
D_{X}=\frac{w}{\pi} \arctan \left(\frac{-X_{Q}}{X_{N}}\right), D_{Y}=\frac{w}{\pi} \arctan \left(\frac{Y_{Q}}{Y_{N}}\right)
$$

\section{Sensor Model with Respect to Harmonic Errors}

Harmonic errors can be caused by various sources, for instance, static misalignment between MP and FP electrodes during system integration, dynamic coupling between the $X-Y$ linear and rotational directions, and external disturbances such as shock and vibration, which affect the sensor system in the form of roll, yaw, and pitch movements.

Those harmonic disturbances are usually minimal. In Fig. 3, yaw movement can be seen as a combination of two separate linear movements in the $X$ and $Y$ directions. Linear movements

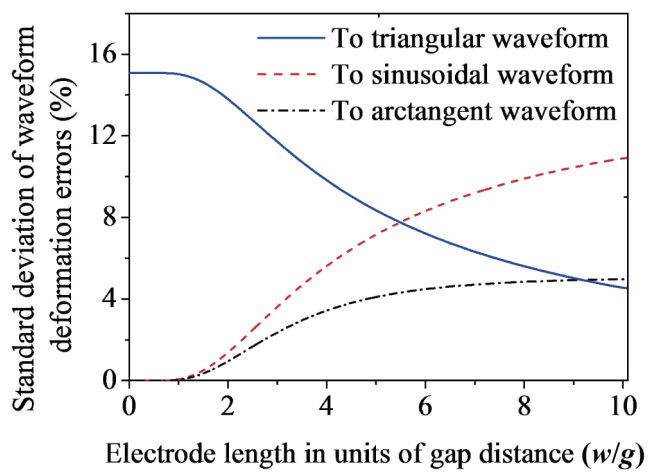

Fig. 2. (Color online) Comparison of standard deviations of waveform deformation errors for different $w / g$ ratios.

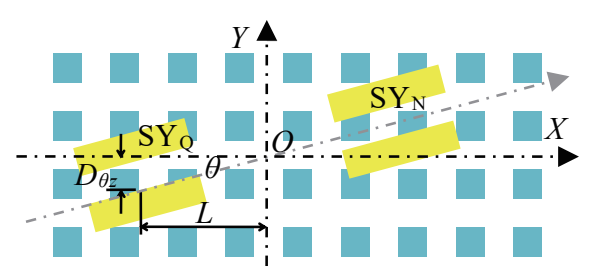

Fig. 3. (Color online) Sensor model considering yaw rotational disturbance. 
of $\mathrm{SX}_{\mathrm{N}}$ and $\mathrm{SX}_{\mathrm{Q}}$ in the $X$ direction cause each to have a phase-shift error as shown in Eq. (3). Similarly, linear movements of $\mathrm{SY}_{\mathrm{N}}$ and $\mathrm{SY}_{\mathrm{Q}}$ in the $Y$ direction cause each to have a phase-shift error as shown in Eq. (4).

$$
\begin{array}{cc}
X_{N}=\cos \left[\pi \frac{\left(D_{X}+D_{\theta Z}\right)}{w}\right] & X_{Q}=-\sin \left[\pi \frac{\left(D_{X}-D_{\theta Z}\right)}{w}\right] \\
Y_{N}=\cos \left[\pi \frac{\left(D_{Y}-D_{\theta Z}\right)}{w}\right] & Y_{Q}=\sin \left[\pi \frac{\left(D_{Y}+D_{\theta Z}\right)}{w}\right] \\
D_{\theta Z}=L \sin \theta_{Z}=L \theta_{Z}
\end{array}
$$

Here, $\theta_{Z}$ is the yaw angle of the MP and $D_{\theta z}$ is the equivalent linear movement of the MP in the measuring direction induced by the yaw angle.

Movements caused by roll and pitch angles can also be considered a combination of a linear movement in the $Z$ direction and another in the $X$ or $Y$ direction as shown in Fig. 4. However, linear movements in the $X$ and $Y$ directions in this study are neglected because they are very small compared with the linear movement in the $Z$ direction. Thus, influences of roll and pitch movements are considered the same as vertical movements, affecting only the amplitude of a sensor signal. Then, the actual signal models against roll, yaw, and pitch disturbances are illustrated by Eqs. (6) and (7).

$$
\begin{array}{ll}
X_{N}^{\prime}=\frac{g}{g+L \theta_{x}} \cos \left[\pi \frac{\left(D_{X}+D_{\theta Z}\right)}{w}\right] & X_{Q}^{\prime}=-\frac{g}{g-L \theta_{x}} \sin \left[\pi \frac{\left(D_{X}-D_{\theta Z}\right)}{w}\right] \\
Y_{N}^{\prime}=\frac{g}{g+L \theta_{y}} \cos \left[\pi \frac{\left(D_{Y}-D_{\theta Z}\right)}{w}\right] & Y_{Q}^{\prime}=-\frac{g}{g-L \theta_{y}} \sin \left[\pi \frac{\left(D_{Y}+D_{\theta Z}\right)}{w}\right]
\end{array}
$$

Under these circumstances, if the actual signal models described by Eqs. (6) and (7) are substituted into Eq. (2) to calculate $X-Y$ displacement of the MP, a harmonic error will be introduced into signal processing, thereby drastically reducing signal linearity.

\section{Five-Dimensional Sensor Prototype System}

Figure 5 presents the schematic structure of the proposed five-dimensional capacitive displacement microsensor system. Common electrodes on the MP are in a similar periodic pattern to those of other capacitive planar positioning systems. However, what is different on the FP is that

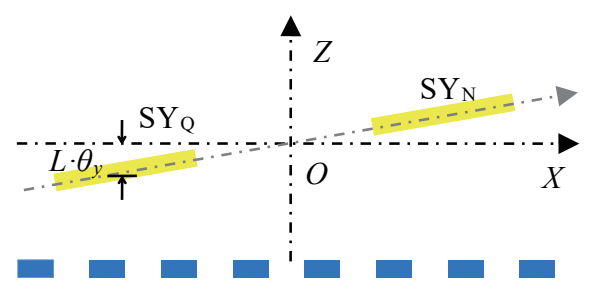

Fig. 4. (Color online) Sensor model considering roll and pitch angular disturbances. 


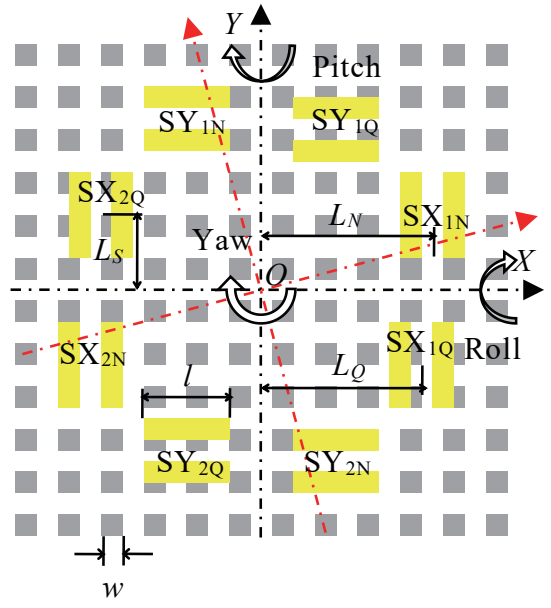

(a)

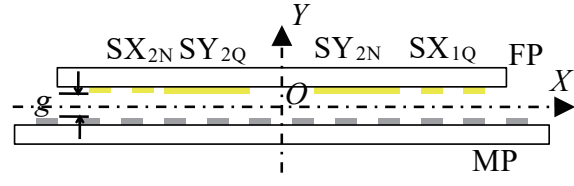

(b)

Fig. 5. (Color online) Schematic views of the proposed capacitive displacement microsensor system: (a) front and (b) side views.

there are eight sensing electrodes on the FP symmetrically positioned around the rotational center $O$ (an assumed origin), namely, $\mathrm{SX}_{1 \mathrm{~N}}, \mathrm{SX}_{1 \mathrm{Q}}, \mathrm{SX}_{2 \mathrm{~N}}, \mathrm{SX}_{2 \mathrm{Q}}, \mathrm{SY}_{1 \mathrm{~N}}, \mathrm{SY}_{1 \mathrm{Q}}, \mathrm{SY}_{2 \mathrm{~N}}$, and $\mathrm{SY}_{2 \mathrm{Q}}$. In the figure, $w$ is the electrode width, $l$ is the sensing electrode length, $L_{N}$ and $L_{Q}$ are the distances of the sensing electrodes $\mathrm{S}_{\mathrm{N}}$ and $\mathrm{S}_{\mathrm{Q}}$ from the rotational center along their measuring direction, respectively, and $L_{S}$ is the distance from the sensing electrodes to the rotational center in a direction perpendicular to the measuring direction.

Based on the analysis results in Sect. 3, we consider the following mathematical signal model of the sensing system in Fig. 5:

$$
\begin{array}{llll}
X_{1 N}^{\prime}=K_{X 1 N} X_{1 N} & X_{2 N}^{\prime}=K_{X 2 N} X_{2 N} & Y_{1 N}^{\prime}=K_{Y 1 N} Y_{1 N} & Y_{2 N}^{\prime}=K_{Y 2 N} Y_{2 N} \\
X_{1 Q}^{\prime}=K_{X 1 Q} X_{1 Q} & X_{2 Q}^{\prime}=K_{X 2 Q} X_{2 Q} & Y_{1 Q}^{\prime}=K_{Y 1 Q} Y_{1 Q} & Y_{2 Q}^{\prime}=K_{Y 2 Q} Y_{2 Q}
\end{array}
$$

where $K_{X 1 N}, K_{X 1 Q}, K_{X 2 N}, K_{X 2 Q}, K_{Y 1 N}, K_{Y 1 Q}, K_{Y 2 N}$, and $K_{Y 2 Q}$ are the approximate relationships of sensor sensitivity to roll and pitch angles, and

$$
\begin{array}{cc}
X_{1 N}=\cos \left[\pi\left(D_{X}+D_{\theta Z}\right) / w\right] & X_{2 N}=\cos \left[\pi\left(D_{X}-D_{\theta Z}\right) / w\right] \\
X_{1 Q}=-\sin \left[\pi\left(D_{X}-D_{\theta Z}\right) / w\right] & X_{2 Q}=\sin \left[\pi\left(D_{X}+D_{\theta Z}\right) / w\right], \\
Y_{1 N}=\cos \left[\pi\left(D_{Y}+D_{\theta Z}\right) / w\right] & Y_{2 N}=\cos \left[\pi\left(D_{Y}-D_{\theta Z}\right) / w\right] \\
Y_{1 Q}=-\sin \left[\pi\left(D_{Y}-D_{\theta Z}\right) / w\right] & Y_{2 Q}=\sin \left[\pi\left(D_{Y}+D_{\theta Z}\right) / w\right], \\
K_{X 1 N}=\frac{g}{g+L_{N} \theta_{y}+L_{S} \theta_{x}} \quad K_{X 1 Q}=\frac{g}{g+L_{Q} \theta_{y}-L_{S} \theta_{x}} \\
K_{X 2 N}=\frac{g}{g-L_{N} \theta_{y}-L_{S} \theta_{x}} K_{X 2 Q}=\frac{g}{g-L_{Q} \theta_{y}+L_{S} \theta_{x}} \\
K_{Y 1 N}=\frac{g}{g+L_{N} \theta_{x}-L_{S} \theta_{y}} K_{Y 1 Q}=\frac{g}{g+L_{Q} \theta_{x}+L_{S} \theta_{y}} \\
K_{Y 2 N}=\frac{g}{g-L_{N} \theta_{x}+L_{S} \theta_{y}} K_{Y 2 Q}=\frac{g}{g-L_{Q} \theta_{x}-L_{S} \theta_{y}} .
\end{array}
$$


By substituting Eq. (8) into Eq. (11), roll and pitch movements are acquired. Their specific values depend closely and only on the amplitude of a readout signal.

$$
\begin{aligned}
& \theta_{x}=\frac{g}{2\left(L_{N}+L_{Q}\right)}\left(\frac{1}{K_{Y 1 N}}+\frac{1}{K_{Y 1 Q}}-\frac{1}{K_{Y 2 N}}-\frac{1}{K_{Y 2 Q}}\right) \\
& \theta_{y}=\frac{g}{2\left(L_{N}+L_{Q}\right)}\left(\frac{1}{K_{X 1 N}}+\frac{1}{K_{X 1 Q}}-\frac{1}{K_{X 2 N}}-\frac{1}{K_{X 2 Q}}\right)
\end{aligned}
$$

Furthermore, by normalizing Eq. (8), impacts of roll and pitch movements are decoupled, resulting in a set of signals containing only $X-Y$ linear movements and yaw movement as in Eqs. (9) and (10). $X-Y$ displacements are obtained by a specific arctangent function as shown in Eq. (13) and, at the same time, yaw movement is determined from Eq. (14). Nominal $X-Y$ movements and yaw movement are decoupled from each completely:

$$
\begin{gathered}
D_{X}=\frac{w}{\pi} \arctan \frac{-X_{1 Q}+X_{2 Q}}{X_{1 N}+X_{2 N}} \quad D_{Y}=\frac{w}{\pi} \arctan \frac{-Y_{1 Q}+Y_{2 Q}}{Y_{1 N}+Y_{2 N}}, \\
\theta_{Z}=\frac{w}{2 \pi L_{S}}\left[\arctan \left(\frac{X_{2 Q}}{X_{1 N}}\right)+\arctan \left(\frac{X_{1 Q}}{X_{2 N}}\right)\right]=\frac{w}{2 \pi L_{S}}\left[\arctan \left(\frac{Y_{2 Q}}{Y_{1 N}}\right)+\arctan \left(\frac{Y_{1 Q}}{Y_{2 N}}\right)\right] .
\end{gathered}
$$

It is also noted that the proposed Eq. (13) considers a set of quadrature signals to calculate MP displacement in both the $X$ and $Y$ directions, which would balance fringe effects. The analyses imply that the waveform nonlinearity of $X-Y$ movement signals caused by rotational disturbances can effectively be eliminated. Also, roll, yaw, and pitch movements can be generated in the proposed scheme.

\section{Experimental Results}

A test bench for the proposed sensor system is constructed as shown in Fig. 6, consisting of a micromotion stage, a vertical motion stage, two tilting motion stages, a rotational motion stage, a capacitive sensor, and an interpolation system. The MP and FP are fabricated as printed circuit boards. A NANOMOTION ${ }^{\circledR}$ motor with a resolution of $10 \mathrm{~nm}$ is applied to provide the displacement of the micro-motion stage. Tilting and rotating stages are mounted to adjust installation errors and system calibration.

From the analysis results in Sect. 2, we concluded that electrode width is a key factor in determining the ratio of sensor waveform nonlinearity to gap distance $(w / g)$. As long as the $w / g$ ratio is less than 2, the actual waveform is closely dependent on sinusoidal waveforms. Minimizing the $w / g$ ratio optimizes signal linearity; however, sensor sensitivity is notably decreased. To keep a balance between signal linearity and sensor sensitivity, the $w / g$ ratio is set to 4 . Figure 7 shows the demodulation outputs of the proposed sensor, namely, $X^{\prime}{ }_{1 N}, X^{\prime}{ }_{2 N}, X^{\prime}{ }_{1 Q}$, and $X_{2 Q}^{\prime}$, in a single period. To better clarify the capability of a five-dimensional displacement measurement, four different sets of rotational inputs are provided in the tests: in set I, the pitch angle $\theta_{y}$ is $0.1^{\circ}$ and the yaw angle $\theta_{z}$ is $0.2^{\circ}$; in set II, the values are 0.2 and $0.4^{\circ}$; and in sets III and IV, the values are 0.4 and $0.8^{\circ}$, and 0.8 and $2.0^{\circ}$, respectively. During all tests, the roll angle $\theta_{x}$ is kept at 0 . As can be seen, rotational disturbances introduce severe sensitivity differences and phase-shift errors into output waveforms. 


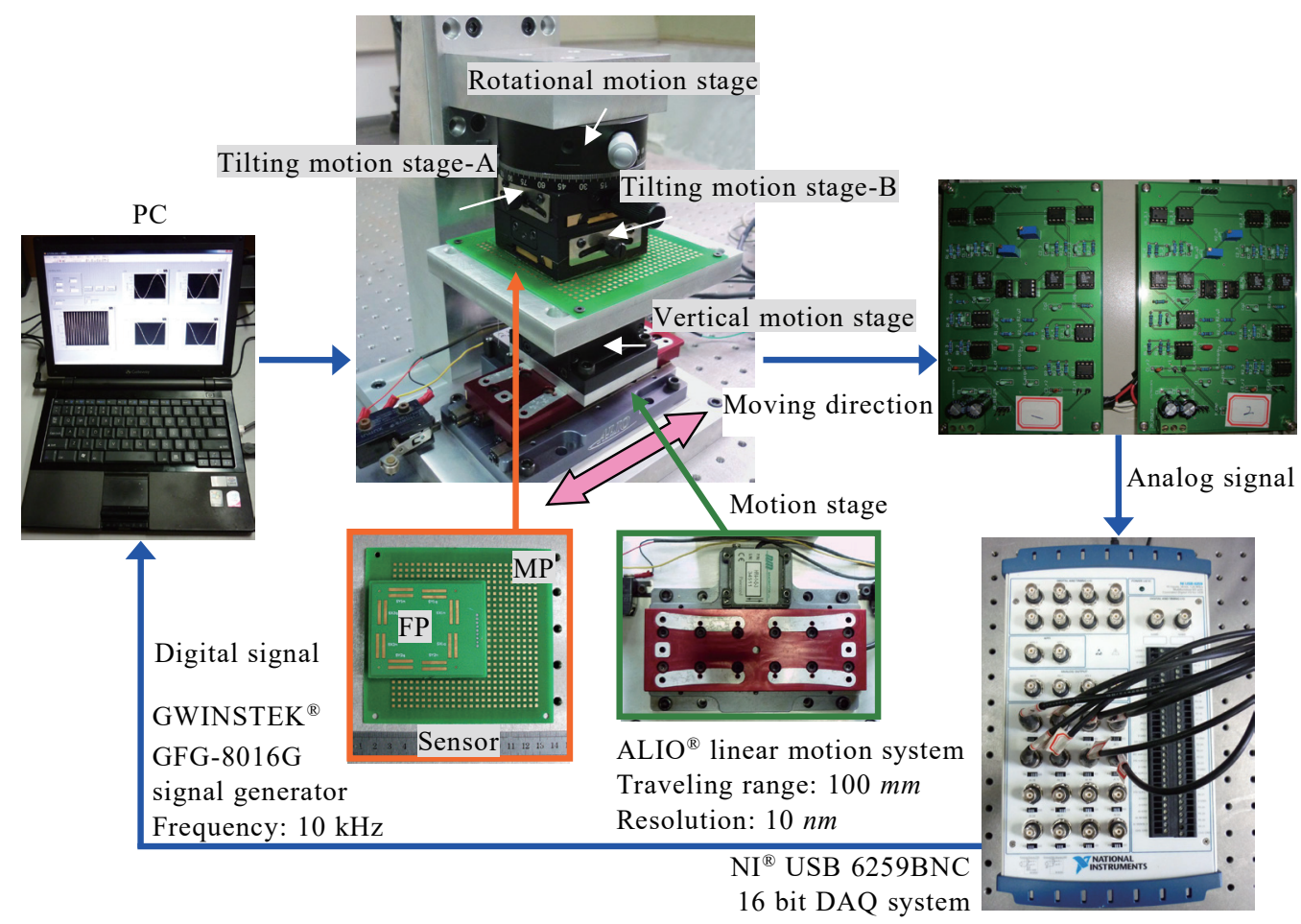

Fig. 6. (Color online) Test bench of the proposed five-dimensional capacitive displacement sensor system.

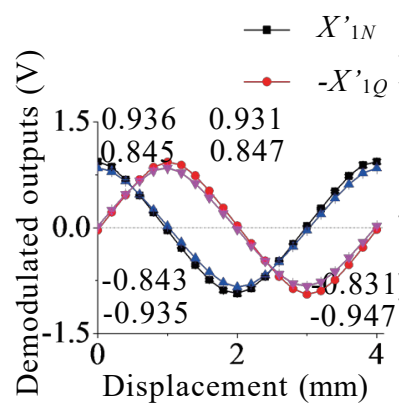

(a)

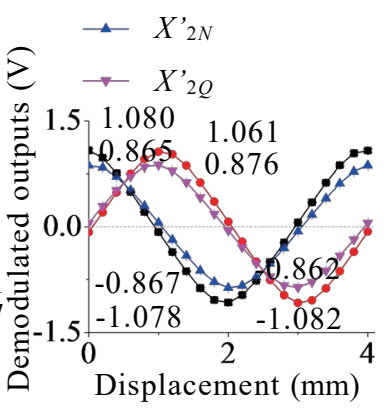

(b)

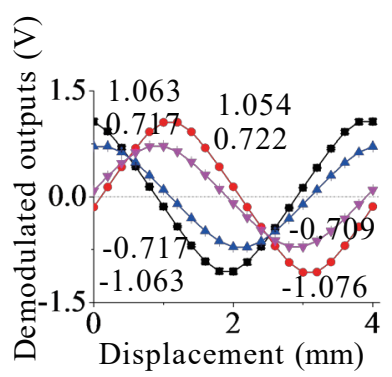

(c)

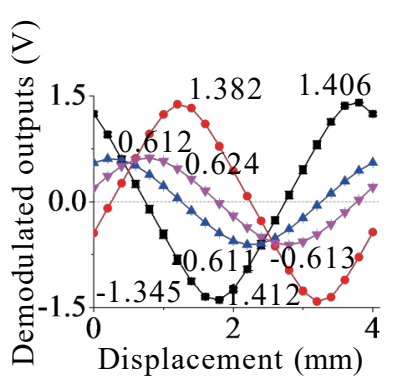

(d)

Fig. 7. (Color online) Demodulated outputs $X^{\prime} 1 N, X^{\prime} 2 N, X^{\prime} 1 Q$, and $X^{\prime} 2 Q$ in a single period: (a) set I, (b) set II, (c) set III, and (d) set IV.

Based on the analysis results in Sect. 3, roll and pitch angles affect signal amplitudes only; therefore, by substituting the amplitudes of the acquired signals into Eq. (12), roll and pitch disturbances can be determined. Figure 8 shows a plot of the decoupled rotational outputs. The calculated results are strongly dependent on ideal inputs. For a $0-0.8^{\circ}$ stroke, roll and pitch angle errors are no larger than $0.01^{\circ}$, which is acceptable for possible closed-loop control purposes.

Figure 9 shows plots of the normalized waveforms $X_{1 N}, X_{2 N}, X_{1 Q}$, and $X_{2 Q}$, with sensitivity differences removed. However, phase-shift errors remain. If acquired normalized waveforms are considered ideal quadrature waveforms, then phase-shift errors diminish measurement accuracy. Figure 10(a) shows a plot of the arctangent waveform directly from Eq. (2). Acquired waveforms exhibit a severe distortion. For sets I to IV, signal nonlinearities range from 1.02 to $6.75 \%$, as yaw 


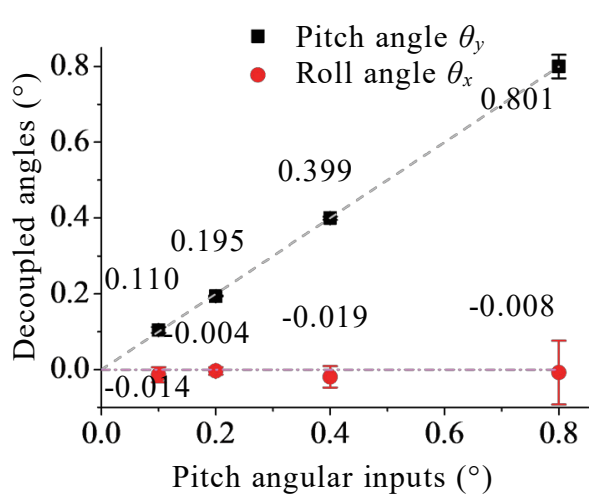

Fig. 8. (Color online) Decoupled roll and pitch rotational outputs.

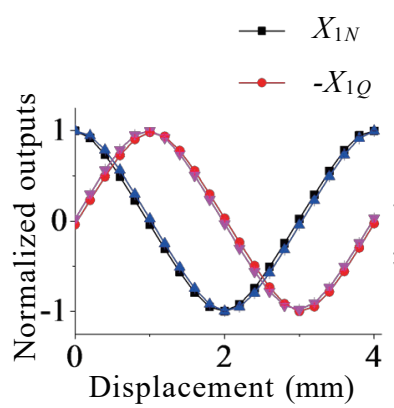

(a)

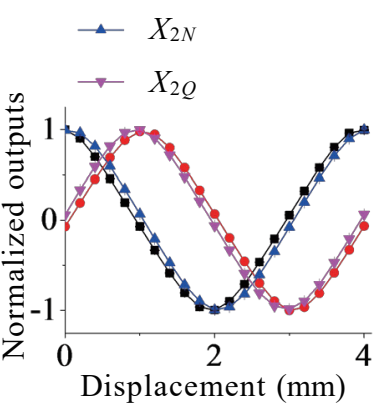

(b)

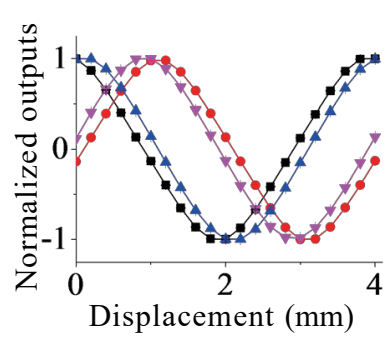

(c)

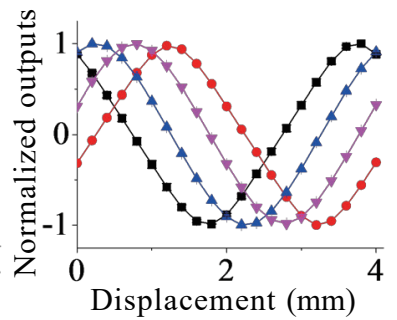

(d)

Fig. 9. (Color online) Normalized outputs $X 1 N, X 2 N, X 1 Q$, and $X 2 Q$ : (a) set I, (b) set II, (c) set III, and (d) set IV.

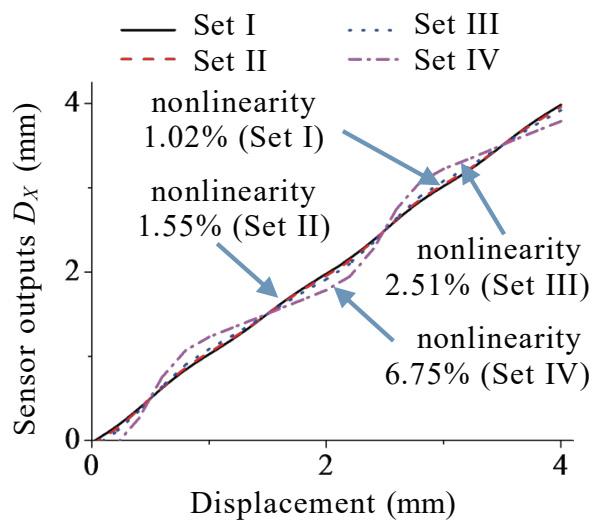

(a)

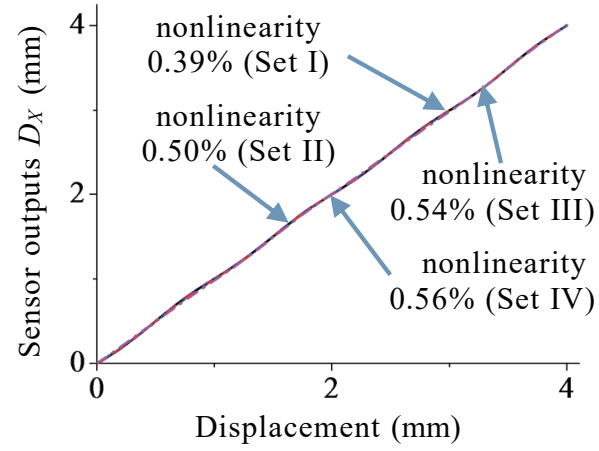

(b)

Fig. 10. (Color online) Acquired nominal displacement in the $X$ direction: (a) conventional and (b) proposed schemes.

inputs increase from 0.2 to $2^{\circ}$. Apparently, Eq. (2) does not meet the requirements for precision measurement, especially in the case of rotational disturbances.

The decoupling method in Eq. (13) removes the influences of phase-shift errors. Figure 10(b) shows the acquired nominal displacement in the $X$ direction. As yaw inputs increase from 0.2 to $2^{\circ}$, signal nonlinearities remain constant, only ranging from 0.39 to $0.56 \%$. This result validates the conclusion regarding Eq. (13). 


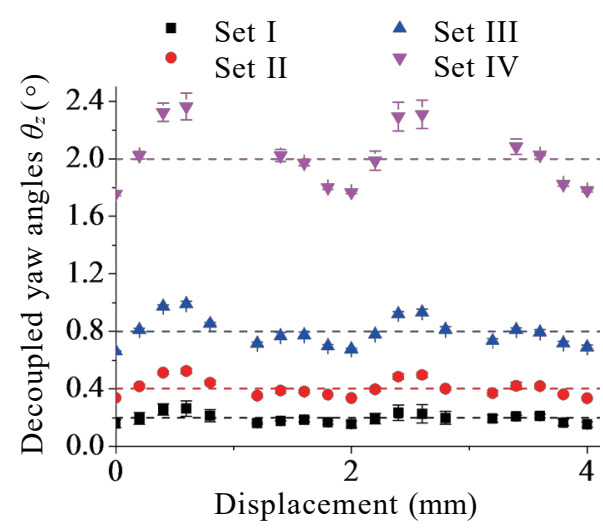

Fig. 11. (Color online) Decoupled yaw angles compared with ideal inputs.
Table 1

Demodulated yaw angles.

Ideal input $\left(^{\circ}\right)$ Average value $\left({ }^{\circ}\right)$ Standard deviation $\left({ }^{\circ}\right)$

$\begin{array}{lll}0.4 & 0.40 & 0.045\end{array}$

$\begin{array}{lll}0.8 & 0.81 & 0.075\end{array}$

$\begin{array}{lll}2.0 & 2.00 & 0.132\end{array}$

Figure 11 shows decoupled yaw angles compared with ideal inputs, the average values of which are listed in Table 1. As can be seen, as the yaw input increases from 0.2 to $2^{\circ}$, the standard deviation of the decoupled output increases as well; more gross errors must be eliminated before the final calculation. The specific reason for this is that signal waveforms are not actually in perfect sinusoidal or cosine model. Nevertheless, the average decoupled yaw angles within a full period are quite cohesive to the ideal inputs, which are no larger than $0.01^{\circ}$.

\section{Conclusions}

In this paper, a novel five-dimensional capacitive displacement sensor is proposed. On the basis of the dataform of the sensor model with respect to harmonic errors, impacts of rotational disturbances on $X-Y$ linear displacement signals are decoupled, and high precision $X_{-}$ $Y$ displacement measurement is achieved, while additional roll, yaw, and pitch movements are acquired. Experiments show that waveform nonlinearities in the $X-Y$ displacement signals are decreased to only $0.5 \%$, while signal errors in the demodulated rotational disturbances are no larger than $0.01^{\circ}$ on a $2^{\circ}$ scale. The advantages of the five-dimensional displacement measurement scheme are validated.

\section{Acknowledgments}

This work was supported by National Natural Science Foundation of China (Grant Nos. 51405263, 51505253, and 51275272).

\section{References}

1 H. J. Ahn and S. Jeon: Mech. Syst. Signal Process. 29 (2012) 148.

2 Y. Saito, Y. Arai, and W. Gao: Sens. Actuators, A 150 (2009) 175.

3 W. Gao, S. Dejima, and S. Kiyono: Sens. Actuators, A 117 (2005) 95.

4 X. H. Huang, J. I. Lee, N. Ramakrishnan, and M. Bedillion: Mechatronics 20 (2010) 27.

5 J. P. Yu, W. Wang, X. Li, and Z. Z. Zhou: Meas. Sci. Rev. 16 (2016) 127. 